\title{
PMD Compensation in Polarization-Multiplexed Multilevel Modulations by Turbo Equalization
}

\author{
Lyubomir L. Minkov, Ivan B. Djordjevic, Lei Xu, and Ting Wang
}

\begin{abstract}
We propose the multilevel maximum a posteriori probability (MAP) detector suitable for use in polarization-multiplexed optical communications using multilevel modulation and coherent detection. The proposed multilevel MAP detection scheme considers two symbols transmitted over two orthogonal polarization states as a two-component symbol. We demonstrate experimentally that the proposed MAP scheme is effective in eliminating the bit-error-ratio (BER) floor phenomenon introduced by the conventional MAP equalizer, which considers the symbols transmitted over both polarizations independently. To improve further tolerance to optical fiber polarization-mode dispersion, we iterate extrinsic soft information between the MAP detector and soft-iterative decoder, in a turbo equalization fashion. Penalty of less than $1.5 \mathrm{~dB}$ at $20 \mathrm{~Gb} / \mathrm{s}$ (and BER of $10^{-6}$ ) for differential group delay of $100 \mathrm{ps}$ is reported.
\end{abstract}

Index Terms-High-speed optical transmission, multilevel modulation, polarization-mode dispersion (PMD), turbo equalization.

\section{INTRODUCTION}

$\mathbf{S}$ ERVICE diversity, network functionalities, and transport capacities of photonics-enabled telecommunications have increased tremendously in recent years. Optical transmission at $100 \mathrm{~Gb} / \mathrm{s}$ per wavelength has been under consideration and planning by network operators. At $100 \mathrm{~Gb} / \mathrm{s}$, optical fiber communications with conventional technologies face the technical challenges of strong signal degradations caused by transmission impairments, such as intra- and inner-channel nonlinearities, the nonlinear phase noise, and polarization-mode dispersion (PMD) [1]-[4]. In order to mitigate the signal distortions at ultrahigh bit rates, some new technologies have been proposed and deployed in optical systems, and they represent a distinctive new trend in optical fiber communications. These new technologies include digital-signal-processing-aided optical channel equalization, digital coherent receiving, multilevel modulations, and optical polarization multiplexing (or optical multiple input multiple output technologies) [1]-[3]. In this letter, we investigate the system performance of a polarization-multiplexed optical communication system using multilevel modulations and coherent detection, and propose new signal processing schemes to further enhance the system performance.

We propose the multilevel maximum a posteriori probability (MAP) turbo equalization (MTE) scheme based on

Manuscript received June 03, 2009; revised July 15, 2009. First published September 09, 2009; current version published November 18, 2009. This work was supported in part by the NSF under Grant IHCS 0725405, and in part by NEC Laboratories America.

L. L. Minkov and I. B. Djordjevic are with the Department of Electrical and Computer Engineering, University of Arizona, Tucson, AZ 85721 USA (e-mail: ljubo@ece.arizona.edu; ivan@ece.arizona.edu).

L. Xu and T. Wang are with NEC Laboratories America, Princeton, NJ 08540 USA (e-mail: leixu@nec-labs.com; ting@nec-labs.com).

Color versions of one or more of the figures in this letter are available online at http://ieeexplore.ieee.org.

Digital Object Identifier 10.1109/LPT.2009.2031087 the multilevel Bahl-Cocke-Jelinek-Raviv (BCJR) algorithm (called here the multilevel BCJR equalizer) and a soft-iterative decoder. This scheme is suitable for PMD compensation in polarization-multiplexed optical communication systems using multilevel modulations and coherent detection. The multilevel BJCR equalizer serves as a nonlinear intersymbol interference equalizer, and provides the soft symbol log-likelihood ratios (LLRs), which are used in soft-decoding process. The unique property of the proposed scheme is that it considers the independent symbols transmitted in two orthogonal polarization states as a super-symbol. The proposed equalizer is able completely to eliminate the error floor phenomenon introduced by a conventional MAP equalizer that considers the symbols transmitted over both polarizations independently. The proposed scheme can be used in combination with an arbitrary soft-decoding scheme. In order to further improve tolerance to PMD, we perform the iteration of extrinsic soft information between a multilevel BCJR equalizer and soft-decoder. We conduct the experimental validation by transmitting two independent binary phase-shift keying (BPSK) signals at 10 $\mathrm{Gb} / \mathrm{s}$ over $H$ - and $V$-polarization channels in the presence of a controlled amount of PMD and amplified spontaneous emission (ASE) noise. We show that in the presence of first-order PMD with a differential group delay (DGD) of 100 ps the optical signal-to-noise ratio (OSNR) penalty, at a bit-error ratio (BER) of $10^{-6}$, is $1.5 \mathrm{~dB}$, which is smaller than PMD penalty found in a nonreturn-to-zero transmission system at $10 \mathrm{~Gb} / \mathrm{s}$ [4].

\section{DESCRIPTION OF Proposed MTE}

Fig. 1 shows the proposed equalizer design. It consists of two parts: 1) a multilevel BCJR equalizer, and 2) a soft-iterative decoder. The multilevel BCJR equalizer [see Fig. 1(a)] uses the independent symbols transmitted in both polarizations as a super-symbol. It operates on an optical channel model implemented in the form of discrete dynamical trellis with memory. The transmitted super-symbol sequence and the received sequences are denoted by $X$ and $Y$, respectively. The super-symbol $x \in \boldsymbol{X}$ has two components $x=\left(s_{H}, s_{V}\right)$, where $s_{H}\left(s_{V}\right)$ denotes the symbol transmitted over horizontal (vertical) polarization. The symbols transmitted over either polarization channel originate from $M$-ary quadrature amplitude modulation (QAM), $M$-ary phase-shift keying (PSK), or $M$-ary pulse-amplitude modulation (PAM), and every symbol carries $\log _{2} M=b$ bits. The received symbol $y \in \boldsymbol{Y}$ has four components $\boldsymbol{y}=\left(\operatorname{Re}\left(y_{H}\right), \operatorname{Im}\left(y_{H}\right), \operatorname{Re}\left(y_{V}\right), \operatorname{Im}\left(y_{V}\right)\right)$, where $\operatorname{Re}\left(y_{H}\right)$ and $\operatorname{Im}\left(y_{H}\right)$ denote the samples corresponding to $I$ and $Q$-channels in horizontal polarization, while $\operatorname{Re}\left(y_{V}\right)$ and $\operatorname{Im}\left(y_{V}\right)$ denote the samples corresponding to $I$ - and $Q$-channels in vertical polarization. $y_{j}$ is the vector of samples that correspond to the transmitted symbol $x_{j}$ for the $j$ th discrete time period. The memory assumption indicates that a super-symbol 


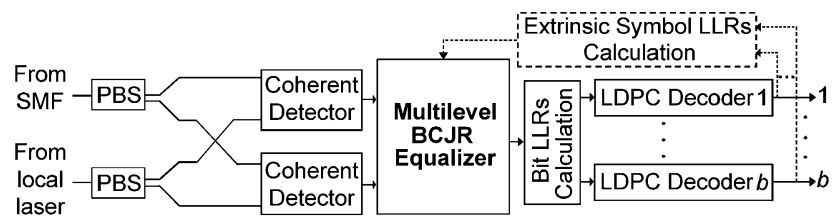

(a)

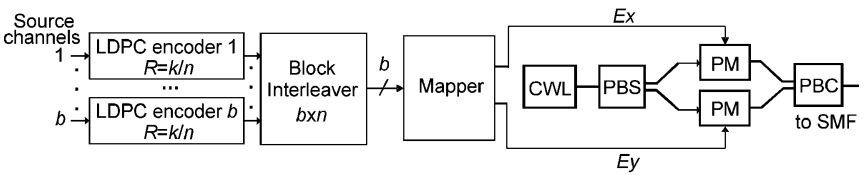

(b)

Fig. 1. Polarization-multiplexed multilevel modulation scheme: (a) receiver and (b) transmitter architectures. CWL: CW laser; PBS/C: polarization beam splitter/combiner; LBC: linear block code; PM: phase modulator.

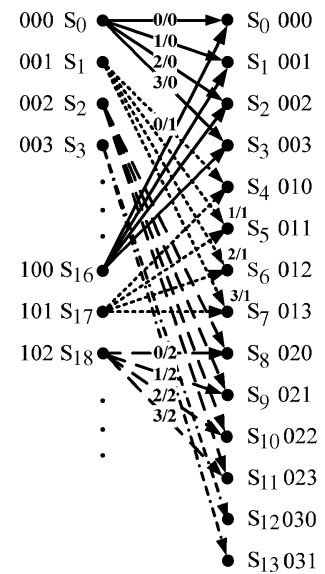

Fig. 2. Four-level trellis example at time instance $j$.

$x_{i}$ is influenced by the preceding $m\left(x_{i-m}, x_{i-m+1}, \ldots, x_{i-1}\right)$ super-symbols and the next $m\left(x_{i+1}, \ldots, x_{i+m}\right)$ super-symbols in the sequence and the memory is denoted by $2 m+1$. A state is defined as $\boldsymbol{s}=\left(x_{i-m}, \ldots, x_{i}, x_{i+1}, \ldots, x_{i+m}\right)$. The trellis is uniquely defined at any moment of time by the triple (previous state, channel output, next state). Fig. 2 illustrates the four-level $(M=4)$ trellis for memory $2 m+1=3$, at time instant $j$. The quaternary numerical system is used for this example (base 4). The left column denotes the previous instance of time, and the right column represents the current instance of time. The trellis has $M^{2 m+1}=64$ states, each corresponding to the different possible three-symbol patterns. $\boldsymbol{S}$ denotes the set of all possible states $\left\{\boldsymbol{s}_{0}, \boldsymbol{s}_{1}, \boldsymbol{s}_{2}, \ldots, \boldsymbol{s}_{63}\right\}$. The middle super-symbol of the current state indicates the output super-symbol, which is to be detected. The branch arrows indicate possible transitions from a given moment in time to the next one. There are two labels above every branch arrow. The first one indicates the transmitted super-symbol and the second one indicates the super-symbol in terminal state. To completely characterize the optical channel, the conditional probability density functions (PDFs) $p\left(y_{j} \mid x_{j}\right)=p\left(y_{j} \mid \boldsymbol{s}\right), \boldsymbol{s} \in \boldsymbol{S}$ are needed. The conditional PDFs are determined by the collecting the histograms by pretransmitting a sufficiently long training sequence. The proposed equalizer operates on the received samples for the $I$ and $Q$ channels and the conditional PDFs. It is universal and applicable to any two-dimensional signal constellation such as $M$-ary PSK, $M$-ary QAM, or $M$-ary PAM, and both coherent and direct detections.
The forward metric is defined as the probability of the terminal state being $\boldsymbol{s}$ given a received sequence $\left(y_{1}, y_{2}, \ldots, y_{j}\right)$. In the log-domain, the corresponding reliability is given by $\alpha_{j}(\boldsymbol{s})=\log \left[p\left(\boldsymbol{s}_{j}=\boldsymbol{s},\left[y_{1} \ldots y_{j}\right]\right)\right]$, $(j=1,2 \ldots, n)$. The backward metric is related to the conditional probability of receiving the next $n-j$ symbols given the current state. In the log-domain, the reliability is defined by $\beta_{j}(\boldsymbol{s})=\log \left[p\left(\left[\boldsymbol{y}_{j+1} \ldots \boldsymbol{y}_{n}\right] \mid \boldsymbol{s}_{j}=\boldsymbol{s}\right)\right]$. The branch metric is defined as the transition probability between two states when a symbol $y_{j}$ has been received. The corresponding reliability in the log-domain is defined by $\gamma_{j}\left(\boldsymbol{s}^{\prime}, \boldsymbol{s}\right)=\log \left[p\left(\boldsymbol{s}_{j}=\boldsymbol{s}, y_{j}, \boldsymbol{s}_{j-1}=\boldsymbol{s}^{\prime}\right)\right]$. These metrics have to be calculated for every discrete period of time as given below

$$
\begin{aligned}
\alpha_{j}(\boldsymbol{s}) & =\max _{\boldsymbol{s}^{\prime}}\left[\alpha_{j-1}\left(\boldsymbol{s}^{\prime}\right)+\gamma_{j}\left(\boldsymbol{s}^{\prime}, \boldsymbol{s}\right)\right] \\
\beta_{j-1}\left(\boldsymbol{s}^{\prime}\right) & =\max _{\boldsymbol{s}}^{*}\left[\beta_{j}(\boldsymbol{s})+\gamma_{j}\left(\boldsymbol{s}^{\prime}, \boldsymbol{s}\right)\right] \\
\gamma_{j}\left(\boldsymbol{s}^{\prime}, \boldsymbol{s}\right) & =\log \left[p\left(y_{j} \mid \boldsymbol{x}[j-m, \ldots, j+m]\right) P\left(x_{j}\right)\right] .
\end{aligned}
$$

The operator max* is defined as $\max ^{*}=\max (x, y)+\log (1+$ $\left.e^{-|x-y|}\right) . P\left(x_{j}\right)$ is the a priori probability of a transmitted symbol $x_{j}$. To estimate the symbol LLRs, we use the following relationship:

$$
\begin{aligned}
\Lambda\left(x_{j}=\delta\right) & =\max _{\left(\boldsymbol{s}^{\prime}, \boldsymbol{s}\right): x_{j}=\delta}\left[\alpha_{j-1}\left(\boldsymbol{s}^{\prime}\right)+\gamma_{j}\left(\boldsymbol{s}^{\prime}, \boldsymbol{s}\right)+\beta_{j}(\boldsymbol{s})\right] \\
& -\max _{\left(\boldsymbol{s}^{\prime}, \boldsymbol{s}\right): x_{j}=\delta_{0}}\left[\alpha_{j-1}\left(\boldsymbol{s}^{\prime}\right)+\gamma_{j}\left(\boldsymbol{s}^{\prime}, \boldsymbol{s}\right)+\beta_{j}(\boldsymbol{s})\right] .
\end{aligned}
$$

Here $\delta$ is the candidate output symbol, and the reliability indicates the likelihood of the received symbol being a particular transmitted symbol $\delta$. For hard decision forward error correction (FEC) schemes, a decision will be performed based on (2) by selecting the symbol with the largest LLR. The use of reference symbol $\delta_{0}=x_{0}$ results in total $M-1$ symbol reliabilities (instead of $M$ ). For soft-decision decoding, the bit reliabilities are calculated in the log domain by observing the binary representation of every symbol. Let $c_{k}$ represent the $k$ th bit in the binary representation of a symbol $x_{j}, k=1,2, \ldots, b$. Then the likelihoods for the bit $c_{k}$ in log domain can be calculated using the already calculated likelihood for all symbols $x_{j}$ as follows:

$$
L\left(\hat{c}_{k}\right)=\log \left[\sum_{x_{j}: c_{k}=0} e^{\left(\Lambda\left(x_{j}\right)\right)}\right]-\log \left[\sum_{x_{j}: c_{k}=1} e^{\left(\Lambda\left(x_{j}\right)\right)}\right] .
$$

Once the bit reliabilities have been calculated, the soft-decoding is performed. Now what is left is to calculate the extrinsic bit reliabilities of soft-decoder, $L_{\text {soft-dec,ext }}\left(c_{k}\right)$, used for improvement of the performance of the multilevel turbo equalizer, which are calculated as the difference between the bit reliabilities of the current and the previous iteration. These extrinsic bit reliabilities are used to calculate the extrinsic symbol reliabilities, which are used as the a priori reliabilities, for subsequent iteration as follows:

$$
L_{\mathrm{BCJR}, \mathrm{apr}}\left(x_{j}\right)=\sum_{k=0}^{l-1}\left(1-c_{k}\right) L_{\mathrm{soft}-\mathrm{dec}, \operatorname{ext}}\left(c_{k}\right)=\log \left[P\left(x_{j}\right)\right] \text {. }
$$

In the rest of this section, we briefly describe the corresponding transmitter shown in Fig. 1(b). The $b$ source bit streams are independently encoded using either a turbo-product code or a low-density parity-check (LDPC) code of rate $R=k / n$, where $k$ is the information word length and $n$ is the 


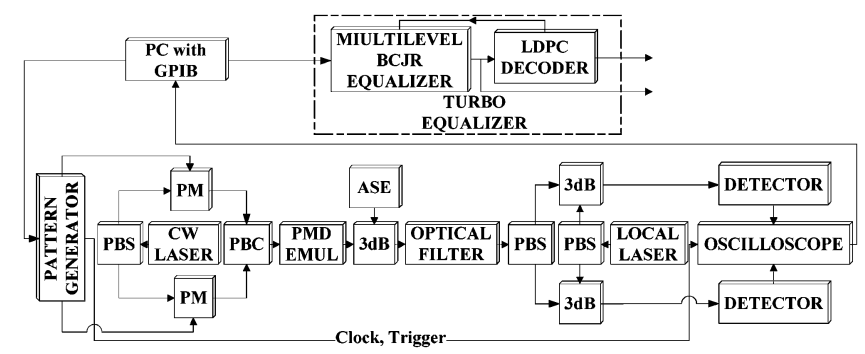

Fig. 3. Experimental setup for polarization multiplexed BPSK. PM: phase modulator; ASE: ASE noise source; $3 \mathrm{~dB}$ : 3-dB coupler.

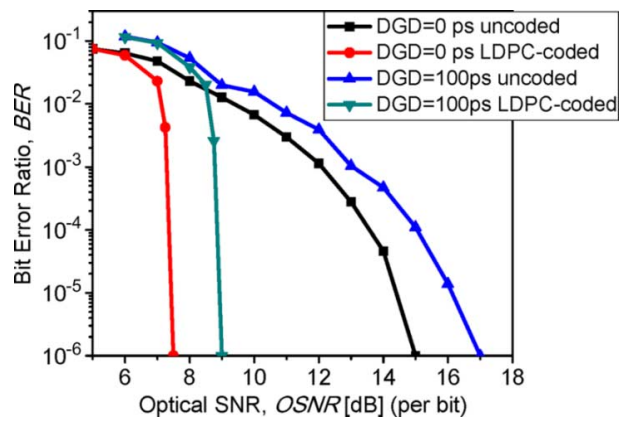

Fig. 4. BER performance of proposed multilevel turbo equalization scheme.



Fig. 5. BER performance of conventional turbo equalization scheme: (a) $\mathrm{DGD}=0 \mathrm{ps}$, and (b) DGD $=100 \mathrm{ps}$.

codeword length. An arbitrary linear block code can be used as shown in Fig. 1(b). At every symbol period time instance, the mapper accepts a vector of $b$ bits from block-interleaver column-wise and determines the constellation points for transmission. In $M$-ary PSK, the outputs of the mapper are used as the modulating signals of the phase modulators (PMs), with $\phi_{l} \in[0,2 \pi / M, 4 \pi / M, \ldots,(M-1) 2 \pi / M], l_{\in}\{H, V\}$. The phase-modulated signals are then combined and transmitted in orthogonal polarizations over the channel.

\section{EXPERIMENTAL SETUP, RESULTS, AND CONCLUSION}

Fig. 3 shows the experimental setup. The two orthogonal polarizations of a continuous-wave laser source are separated by a polarization beam splitter and are modulated by two PMs (Covega) driven at $10 \mathrm{~Gb} / \mathrm{s}$ (Anritsu MP1763C). A precoded test pattern was loaded into the pattern generator via a personal computer (PC) with general purpose interface bus (GPIB) interface. A polarization beam combiner was used to combine the two modulated signals, followed by a PMD emulator (JDSU PE3) which introduced a controlled amount of DGD to the signal. Then the signal distorted by PMD was mixed with a controlled amount of ASE noise with a 3-dB coupler. A modulated signal level was maintained at $0 \mathrm{~dB}$ while the ASE power level was changed to obtain different OSNRs. Next, the optical signal was preamplified, filtered (JSDU $2 \mathrm{~nm}$ bandpass filter), and coher- ently detected. The coherent detection is performed by mixing the received signal with the signal from the local laser with a $3-\mathrm{dB}$ coupler. The resulting signal is detected with a detector (Agilent 11982A) and an oscilloscope (Agilent DCA 86105A), triggered by the data pattern that was used to acquire the samples. To maintain a constant power of $-6 \mathrm{dBm}$ at the detector, a variable attenuator was used. Data was transferred via GPIB back to the PC. The PC also served as a multilevel turbo equalizer with offline processing. A pattern length of 10 million bits was used for measurements at BER $=10^{-6}$.

The experimental results for BER performance of the proposed multilevel turbo equalizer are summarized in Fig. 4. For the experiment, a regular quasi-cyclic LDPC $(16935,13550)$ code of girth 10 and column weight 3 was used as channel code. It has been shown that this code does not exhibit error floor phenomena down to BER $=10^{-9}$. The number of extrinsic iterations between the LDPC decoder and BCJR equalizer was set to 3, and the number of the intrinsic LDPC decoder iterations was set to 25 . The state memory of $2 m+1=3$ was sufficient for the compensation of the first-order PMD with DGD of 100 ps. The OSNR penalty for 100 ps of DGD is $1.5 \mathrm{~dB}$ at a BER of $10^{-6}$. Coding gain for a DGD of $0 \mathrm{ps}$ is $7.5 \mathrm{~dB}$ at a BER of $10^{-6}$, and the coding gain for a DGD of $100 \mathrm{ps}$ is $8 \mathrm{~dB}$. The BER performance, when polarization channels were considered independently, for a DGD of 0 ps, is shown in Fig. 5(a), while that for a DGD of 100 ps is shown in Fig. 5(b). The uncoded curves for a DGD of 100 ps exhibit a BER floor, which is so high that the use of advanced FEC is necessary. Using a hard decision equalizer would result in an error floor while the proposed equalization scheme eliminates the error floor and allows for implementation of FEC codes other than LDPC codes. The proposed multilevel turbo equalization scheme outperforms the corresponding scheme that considers the polarization channels independently by even $5 \mathrm{~dB}$ at BER of $10^{-6}$ for DGD of $100 \mathrm{ps}$ and by $5.5 \mathrm{~dB}$ at BER of $10^{-6}$ for DGD of $0 \mathrm{ps}$.

In conclusion, we proposed a particular turbo equalization that considers independent symbols transmitted over both polarizations as a super-symbol. This scheme is suitable for PMD compensation in polarization-multiplexed multilevel modulation schemes with coherent detection. We have shown that the proposed equalization scheme can significantly improve the performance of the system by compensating for any imbalance in polarization and phase. The improvement of the proposed turbo equalizer over a conventional one, for DGD of 100 ps at BER of $10^{-6}$, is $5 \mathrm{~dB}$. The proposed approach is universal and can be applied to arbitrary multilevel modulation schemes. By approximating $\max ^{*}(x, y)$ by $\max (x, y)$, the forward and backward steps in BCJR equalizer become Viterbi equalizers, which are already implemented at $10 \mathrm{~Gb} / \mathrm{s}$.

\section{REFERENCES}

[1] S. Walklin and J. Conradi, "Multilevel signaling for increasing the reach of $10 \mathrm{~Gb} / \mathrm{s}$ lightwave systems," J. Lightw. Technol., vol. 17, no. 11, pp. 2235-2248, Nov. 1999.

[2] J. Renaudier, G. Charlet, M. Salsi, O. B. Pardo, H. Mardoyan, P. Tran, and S. Bigo, "Linear fiber impairments mitigation of $40-\mathrm{Gbit} / \mathrm{s}$ polarization-multiplexed QPSK by digital processing in a coherent receiver," J. Lightw. Technol., vol. 26, no. 1, pp. 36-42, Jan. 1, 2008.

[3] G. Colavolpe, T. Foggi, E. Forestieri, and G. Prati, "Multilevel optical systems with MLSD receivers insensitive to GVD and PMD," $J$. Lightw. Technol., vol. 26, no. 10, pp. 1263-1273, May 15, 2008.

[4] L. L. Minkov, I. B. Djordjevic, L. Xu, T. Wang, and F. Kueppers, "Evaluation of large girth LDPC codes for PMD compensation by turbo equalization," Opt. Express, vol. 16, pp. 13450-13455, 2008. 